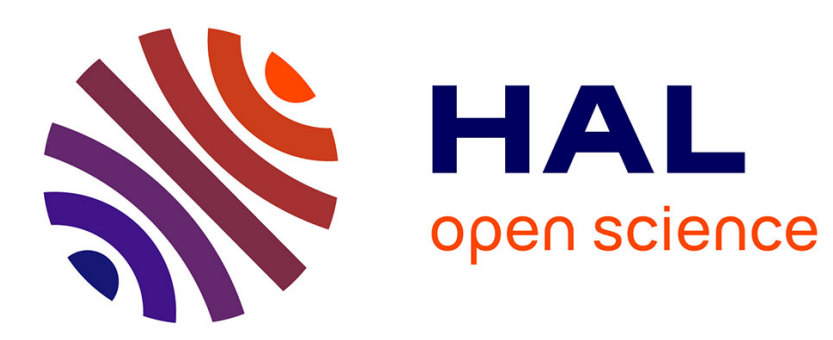

\title{
The Goh necessary optimality conditions for the Mayer problem with control constraints
}

Hélène Frankowska, Daniela Tonon

\section{To cite this version:}

Hélène Frankowska, Daniela Tonon. The Goh necessary optimality conditions for the Mayer problem with control constraints. Decision and Control, 2013, pp.538-543. hal-01067270

\section{HAL Id: hal-01067270 \\ https://hal.science/hal-01067270}

Submitted on 23 Sep 2014

HAL is a multi-disciplinary open access archive for the deposit and dissemination of scientific research documents, whether they are published or not. The documents may come from teaching and research institutions in France or abroad, or from public or private research centers.
L'archive ouverte pluridisciplinaire HAL, est destinée au dépôt et à la diffusion de documents scientifiques de niveau recherche, publiés ou non, émanant des établissements d'enseignement et de recherche français ou étrangers, des laboratoires publics ou privés. 


\title{
The Goh necessary optimality conditions for the Mayer problem with control constraints
}

\author{
Hélène Frankowska and Daniela Tonon*
}

\begin{abstract}
The well known Goh second order necessary optimality conditions in optimal control theory concern singular optimal controls taking values in the interior of a set of controls $U$. In this paper we investigate these conditions for the Mayer problem when $U$ is a convex polytope or a closed subset of class $C^{2}$ for an integrable optimal control $\bar{u}(\cdot)$ that may take values in the boundary of $U$. This is indeed a frequent situation in optimal control and for this reason the understanding of this issue is crucial for the theory of second order optimality conditions. Applying the Goh transformation we derive necessary conditions on tangent subspace to $U$ at $\bar{u}(t)$ for almost all t's.

In the presence of an endpoint constraint, if the Mayer problem is calm, then similar second order necessary optimality conditions are satisfied whenever the maximum principle is abnormal. If it is normal, then analogous results hold true on some smaller subspaces.
\end{abstract}

\section{Introduction}

Let $U \subset \mathbb{R}^{m}, K \subset \mathbb{R}^{n}$ be closed, $x_{0} \in \mathbb{R}^{n}, f:[0,1] \times$ $\mathbb{R}^{n} \times \mathbb{R}^{m} \rightarrow \mathbb{R}^{n}$ and $\varphi: \mathbb{R}^{n} \rightarrow \mathbb{R}$ be sufficiently regular. Consider the Mayer optimal control problem

$$
\min \left\{\varphi(x(1)): x(\cdot) \in S_{[0,1]}\left(x_{0}\right), x(1) \in K\right\},
$$

where $S_{[0,1]}\left(x_{0}\right)$ is the set of all absolutely continuous solutions of the control system

$$
x^{\prime}(t)=f(t, x(t), u(t)), u(t) \in U, x(0)=x_{0} .
$$

${ }^{*}$ This work was co-funded by the European Union under the 7 th Framework Programme "FP7-PEOPLE-2010-ITN", grant agreement number 264735-SADCO.

${ }^{\dagger}$ Hélène Frankowska is with Institut de Mathématiques de Jussieu, Université Pierre et Marie Curie, 4 place Jussieu, 75252 Paris, France frankowska@math. jussieu.fr

${ }^{\ddagger}$ Daniela Tonon is with Department of Electrical and Electronic Engineering, Imperial College London, Exhibition Road, London, SW7 2BT, UK, dtonon@imperial.ac.uk
Define the Hamiltonian $\mathscr{H}:[0,1] \times \mathbb{R}^{n} \times \mathbb{R}^{n} \times \mathbb{R}^{m} \rightarrow$ $\mathbb{R}$ :

$$
\mathscr{H}(t, x, p, u)=\langle p, f(t, x, u)\rangle .
$$

We first consider the Mayer problem without endpoint constraints:

$$
\min \left\{\varphi(x(1)): x(\cdot) \in S_{[0,1]}\left(x_{0}\right)\right\} .
$$

Let $(\bar{x}(\cdot), \bar{u}(\cdot))$ be a strong local minimizer of (3). By the maximum principle, for an absolutely continuous $p:[0,1] \rightarrow \mathbb{R}^{n}$ satisfying

$$
-p^{\prime}(t)=\mathscr{H}_{x}(t, \bar{x}(t), p(t), \bar{u}(t)) \text { for a.e. } t \in[0,1]
$$

and

$$
-p(1)=\nabla \varphi(\bar{x}(1))
$$

the following maximum principle holds true for a.e. $t$ :

$$
\langle p(t), f(t, \bar{x}(t), \bar{u}(t))\rangle=\max _{u \in U}\langle p(t), f(t, \bar{x}(t), u)\rangle .
$$

Thus for a.e. $t \in[0,1]$ such that the optimal control $\bar{u}(t)$ lies in the interior of $U, \mathscr{H}_{u}[t]=0$ and the LegendreClebsch condition $\mathscr{H}_{u u}[t] \leq 0$ is satisfied, where $[t]:=$ $(t, \bar{x}(t), p(t), \bar{u}(t))$. If $\bar{u}(\cdot)$ is singular, in the sense that $\mathscr{H}_{u u}[\cdot]=0$, the Legendre-Clebsch condition doesn't bring any additional information about optimal controls. Different, but related, definitions of singular controls and discussions on their importance can be found for instance in $[1,2,3]$, and the references therein.

Assume that $\mathscr{H}_{u u}[t]=0$ a.e. in $\left[t_{1}, t_{2}\right]$. According to [4], if $U$ is an open convex polytope, then the matrix $\mathscr{H}_{x u}[t] f_{u}[t]$ is symmetric and

$$
\begin{aligned}
R(t):= & \frac{1}{2} f_{u}[t]^{*} \mathscr{H}_{x x}[t] f_{u}[t]-\frac{1}{2} \frac{d}{d t} \mathscr{H}_{x u}[t] f_{u}[t] \\
& -\mathscr{H}_{x u}[t] f_{x}[t] f_{u}[t]+\frac{1}{2} \mathscr{H}_{x u}[t] \frac{d}{d t} f_{u}[t]
\end{aligned}
$$

is negative definite for a.e. $t \in\left[t_{1}, t_{2}\right]$. The first property, known in the literature as the Goh condition, in the case of affine control systems reduces to a condition on the Lie brackets of the flux functions, as we recall 
in Remark 3.2. The second property can be seen as a generalized Legendre-Clebsch condition.

To deal with closed control constraints Goh also suggested to use variations of the optimal control which are equal to zero when $\bar{u}(t) \in \partial U$. However, this can't be applied to recover second-order optimality conditions when $\bar{u}(t) \in \partial U$ almost everywhere.

We present here a different approach, based on non-zero variations of the optimal control even when $\bar{u}(\cdot)$ is a boundary control.

For every $u \in U$ let us consider the maximal subspace $P_{u}$ contained in the tangent cone to $U$ at $u$.

Below, Theorems 4.1 and 5.2 provide pointwise second order necessary optimality conditions respectively in the case when $U$ is a convex polytope or a subset of class $C^{2}$ for a singular optimal control $\bar{u}(\cdot)$ being merely integrable. Considering $\mathscr{H}_{x u}[t] f_{u}[t]$ and $R(t)$ as bilinear operators on the subspace $P_{\bar{u}(t)}$, in both cases, we still have symmetry of $\mathscr{H}_{x u}[t] f_{u}[t]$. In the former case we also get the negative definiteness of $R(t)$ on $P_{\bar{u}(t)}$.

In Corollary 4.2 we prove that if $U$ is a closed convex polytope in $\mathbb{R}^{m}$ and $f$ is affine with respect to $u$ :

$$
f(x, u)=f_{0}(x)+g(x) u,
$$

with $g(\cdot) \in C^{2}\left(\mathbb{R}^{n} ; M(n \times m)\right.$ and if $g_{k}(x)$ denotes the $k$-th column of the matrix $g(x)$, then the matrix valued mapping $\Theta:[0,1] \rightarrow M(m \times m)$, given by

$$
\theta_{k, l}(t):=\left\langle p(t),\left[g_{k}, g_{l}\right](\bar{x}(t))\right\rangle \quad \forall k, l \in\{1, \ldots, m\},
$$

enjoys the following property: for a.e. $t \in[0,1]$, the subspace $\Theta(t)\left(P_{\bar{u}(t)}\right)$ (the image of $P_{\bar{u}(t)}$ by the linear operator $\Theta(t))$ is orthogonal to the subspace $P_{\bar{u}(t)}$. In particular we recover the well known Goh condition in the geometric optimal control: $\Theta(t)=0$ for a.e. $t$ such that $\bar{u}(t) \in \operatorname{Int} U$.

For the Mayer problem with the endpoint constraint $K$, we assume calmness at $\bar{x}(\cdot)$. This allows to reduce the problem to the one without endpoint constraints but involving a nondifferentiable cost function and so the classical first and second order necessary conditions are not satisfied. Still we are able to derive second order conditions through a variational approach similar to the one without end point constraints.

Since the very first work by Goh, [4], the Goh condition was investigated by many researchers in the geometric optimal control theory following fundamental works by Krener, cf. [5] and [6], on the Goh condition (and its higher order extensions) when the optimal control is piecewise $C^{\infty}$. His approach was taken over by several authors and the Goh condition became mostly known in the form involving Lie brackets, as a second-order necessary optimality condition for the abnormal maximum principle. See for instance $[7,8]$ and [3, pp.314-319].

Several authors made an extensive use of the Goh transformation to recover necessary and sufficient second order optimality conditions in an integral and a pointwise form, see for instance [9], [10] and the bibliographies contained therein. However, in these latter works many additional structural restrictions on the control system, control sets and also on the optimal control are imposed, as for instance in Assumptions 1, 2 from [9] concerning the structure optimal controls .

The outline of this paper is as follows. In Section 2 we introduce some notations and provide some preliminary results. Section 3 is devoted to the second order necessary optimality conditions for an arbitrary set $U$ and any integrable optimal control at times when it belongs to the interior of $U$. Section 4 deals with the case when $U$ is a convex polytope and Section 5 with the case when $U$ is of class $C^{2}$. The last section adresses the second order necessary optimality conditions for the Mayer problem involving endpoint constraints.

Most of results presented here are given without proofs, because they are quite long and technical and will be published elsewhere.

\section{Preliminaries and notations}

The norm in $\mathbb{R}^{n}$ is denoted by $\|\cdot\|$ and the inner product by $\langle\cdot, \cdot\rangle$. Let $\stackrel{\circ}{B}:=\left\{x \in \mathbb{R}^{n}:\|x\|<1\right\}$ be the open unit ball in $\mathbb{R}^{n}$. For a nonempty subset $K$ of $\mathbb{R}^{n}$, $\partial K$ denotes its boundary, Int $K$ its interior and $K^{c}$ its complement. The distance function $d_{K}: \mathbb{R}^{n} \rightarrow \mathbb{R}_{+}$is defined by $d_{K}(x):=\inf _{y \in K}\|x-y\|$ for all $x \in \mathbb{R}^{n}$ and the oriented distance $b_{K}: \mathbb{R}^{n} \rightarrow \mathbb{R}$ from $K \neq \mathbb{R}^{n}$ by $b_{K}(x):=d_{K}(x)-d_{K^{c}}(x)$ for all $x \in \mathbb{R}^{n}$. We set $b_{K}(\cdot)=0$ if $K=\mathbb{R}^{n}$. Finally, by $M(k \times r)$ we denote the space of matrices having $k$ lines and $r$ columns.

Definition 2.1 Let $C: \mathbb{R}^{m} \rightarrow \mathbb{R}^{m}$ be a linear mapping and $F$ be a subspace of $\mathbb{R}^{m}$. We say that $C$ is symmetric on $F$ if $\langle C u, v\rangle=\langle u, C v\rangle$ for all $u, v \in F$ and that $C$ is negative definite on $F$ if $\langle C u, u\rangle \leq 0$ for all $u \in F$.

Let $U$ be a nonempty closed subset of $\mathbb{R}^{m}$. For $u_{0} \in$ $U$ the adjacent tangent cone to $U$ at $u_{0}$ is defined by

$$
T_{U}^{b}\left(u_{0}\right):=\left\{u \in \mathbb{R}^{n}: \lim _{h \rightarrow 0+} \frac{d_{U}\left(u_{0}+h u\right)}{h}=0\right\} .
$$

The second-order adjacent subset to $U$ at $\left(u_{0}, u\right) \in$ $\mathbb{R}^{m} \times \mathbb{R}^{m}$ is the set defined by

$T_{U}^{b(2)}\left(u_{0}, u\right):=\left\{v \in \mathbb{R}^{n}: \lim _{h \rightarrow 0+} \frac{d_{U}\left(u_{0}+h u+h^{2} v\right)}{h^{2}}=0\right\}$. 
For any $u \in U$ we denote by

$P_{u}$ the largest subspace contained in $T_{U}^{b}(u)$.

Let $f:[0,1] \times \mathbb{R}^{n} \times \mathbb{R}^{m} \rightarrow \mathbb{R}^{n}, f^{\prime}(t, x, u)$ be the derivative of $f(t, \cdot, \cdot)$ at $(x, u), f_{x}(t, x, u)$ and $f_{u}(t, x, u)$ be the partial derivatives of $f$ with respect to $x$ and $u ; f^{\prime \prime}(t, x, u)$ denotes the Hessian of $f(t, \cdot, \cdot)$ at $(x, u)$, while $f_{x x}, f_{x u}$ and $f_{u и}$ denote the second-order partial derivative of $f$ with respect to $x$ and $u$.

Fix a trajectory-control pair $(\bar{x}(\cdot), \bar{u}(\cdot))$ of (2) and set $f[t]=f(t, \bar{x}(t), \bar{u}(t))$. Let $f_{u}[t], f_{x}[t]$ and $f^{\prime \prime}[t]$ be defined in a similar way. Throughout the whole paper we impose the following assumptions : For some $\delta>0$

(a) $f(t, \cdot, \cdot) \in C^{2}$ on $(\bar{x}(t)+\delta \stackrel{\circ}{B}) \times(\bar{u}(t)+\delta \stackrel{\circ}{B}), \forall t$;

(b) $\left\|f_{u}[\cdot]\right\|, \quad\left\|f^{\prime}[\cdot]\right\|, \quad\left\|f^{\prime \prime}[\cdot]\right\| \in L^{1}\left([0,1] ; \mathbb{R}_{+}\right)$and $\exists a(\cdot) \in L^{1}\left([0,1] ; \mathbb{R}_{+}\right)$such that the mappings $f^{\prime}(t, \cdot, \cdot)$ and $f^{\prime \prime}(t, \cdot, \cdot)$ are $a(t)$-Lipschitz on $(\bar{x}(t)+\delta \stackrel{\circ}{B}) \times(\bar{u}(t)+\delta \stackrel{\circ}{B})$ for a.e. $t \in[0,1] ;$

(c) $f(t, x, U)$ is closed for all $t \in[0,1], x \in \bar{x}(t)+\delta \stackrel{8}{B}$;

(d) $\varphi(\cdot) \in C^{2}$ on $\bar{x}(1)+\delta \stackrel{\circ}{ }$.

Recall that $(\bar{x}(\cdot), \bar{u}(\cdot))$ is a strong local minimizer for (3) (resp. (1)) if there exists $\eta>0$ such that $\bar{x}(\cdot)$ minimizes $\varphi$ over all $x(\cdot) \in S_{[0,1]}\left(x_{0}\right)$ (resp. over all $x(\cdot) \in S_{[0,1]}\left(x_{0}\right)$ with $x(1) \in K$ ) satisfying $\|\bar{x}(\cdot)-x(\cdot)\|_{\infty} \leq \eta$.

Remark 2.2 Results contained in Sections 3-5 below are stated only for strong local minimizers. However they are also valid for the so called weak local minimizers. In contrast, Section 6 concerns only strong local minimizers. Not to switch between these different notions, in this paper we investigate only strong local minimizers.

Theorem 2.3 (Maximum Principle, [11]) Let $(\bar{x}(\cdot), \bar{u}(\cdot))$ be a strong local minimizer of the Mayer problem (3). Then there exists an absolutely continuous $p:[0,1] \rightarrow \mathbb{R}^{n}$ satisfying (4), (5) and (6). Furthermore, for a.e. $t \in[0,1]$ and for any $u \in P_{\bar{u}(t)}$ we have $\mathscr{H}_{u}[t] u=0$.

Define $A:=\left\{t \in[0,1]: \bar{u}(t) \in \partial U, \mathscr{H}_{u}[t] \neq 0\right\}$ and

$$
\begin{aligned}
\mathscr{U}_{A} & =\left\{u(\cdot) \in L^{\infty}\left([0,1] ; \mathbb{R}^{m}\right): \exists c>0, h_{0}>0\right. \text { s.t. } \\
& \left.\forall h \in\left[0, h_{0}\right], \forall t \in A, d_{U}(\bar{u}(t)+h u(t)) \leq c h^{2}\right\} .
\end{aligned}
$$

Note that for any $u(\cdot) \in \mathscr{U}_{A}, u(t) \in T_{U}^{b}(\bar{u}(t))$ a.e. in $A$.

\section{Theorem 2.4 (Integral second order conditions)}

Consider a strong local minimizer $(\bar{x}(\cdot), \bar{u}(\cdot))$ of the Mayer problem (3). Then for all $u(\cdot), \quad v(\cdot) \in L^{\infty}\left([0,1] ; \mathbb{R}^{m}\right)$ such that $u(\cdot) \in \mathscr{U}_{A}$ and $v(t) \in T_{U}^{b(2)}(\bar{u}(t), u(t))$ for a.e. $t \in A$, and for $p(\cdot)$ as in the maximum principle either $\int_{0}^{1} \mathscr{H}_{u}[t] u(t) d t<0$ or $\mathscr{H}_{u}[t] u(t)=0$ a.e. in $[0,1]$ and

$$
\begin{aligned}
0 \geq \quad & \int_{0}^{1}\left(\mathscr{H}_{u}[t] v(t)+\frac{1}{2} y(t)^{*} \mathscr{H}_{x x}[t] y(t)\right. \\
& \left.+u(t)^{*} \mathscr{H}_{x u}[t] y(t)+\frac{1}{2} u(t)^{*} \mathscr{H}_{u u}[t] u(t)\right) d t \\
& -\frac{1}{2} y(1)^{*} \varphi^{\prime \prime}(\bar{x}(1)) y(1),
\end{aligned}
$$

where $y(\cdot)$ solves the linear system

$$
\left\{\begin{array}{l}
y^{\prime}(t)=f_{x}[t] y(t)+f_{u}[t] u(t) \\
y(0)=0 .
\end{array}\right.
$$

The proof follows the same lines as the one in [12].

\section{Second order conditions in the interior of control constraints}

Consider a strong local minimizer $(\bar{x}(\cdot), \bar{u}(\cdot))$ of the Mayer problem (3) and $p(\cdot)$ as in the maximum principle of Theorem 2.3. Let the matrix valued mapping $R(t)$ be defined as in the introduction.

Theorem 3.1 Assume that for some $0 \leq t_{1}<t_{2} \leq 1$, $\bar{u}(\cdot)$ is integrable on $\left[t_{1}, t_{2}\right]$ and

$$
\begin{aligned}
& \mathscr{H}_{u u}[t]=0 \text { a.e. in }\left[t_{1}, t_{2}\right] \\
& \mathscr{H}_{x u}[\cdot], f_{u}[\cdot] \text { are Lipschitz on }\left[t_{1}, t_{2}\right] ; \\
& \mathscr{H}_{x x}[\cdot], f_{x}[\cdot] \text { are essentially bounded on }\left[t_{1}, t_{2}\right] .
\end{aligned}
$$

Then, for a.e. $t \in\left[t_{1}, t_{2}\right]$ such that $\bar{u}(t) \in$ Int $U$, the matrix $\mathscr{H}_{x u}[t] f_{u}[t]$ is symmetric and $R(t) \leq 0$.

The statement of the above theorem is proved for the Lebesgue points $t$ of $(\bar{u}(\cdot), R(\cdot))$ such that $\bar{u}(t) \in \operatorname{Int} U$. The proof is long and technical and will be published elsewhere. It involves the Goh transformation and some advanced properties of Lebesgue points.

Remark 3.2 If $f$ is affine with respect to controls, the symmetry of the matrix $\mathscr{H}_{x u}[t] f_{u}[t]$ implies that the adjoint state is orthogonal to the Lie brackets of the flux. Indeed let $f$ be as in (7), where $g(\cdot) \in C^{2}\left(\mathbb{R}^{n} ; M(n \times m)\right)$ and $U$ is a closed nonempty subset of $\mathbb{R}^{m}$ and let $g_{k}(x)$ denote the $k$-th column of $g(x)$. Then for all $t$ such that $\mathscr{H}_{x u}[t] f_{u}[t]$ is symmetric and for any $k, l \in\{1, \ldots, m\}$,

$$
\begin{aligned}
0 & =\left(\mathscr{H}_{x u}[t] f_{u}[t]-f_{u}[t]^{*} \mathscr{H}_{x u}[t]^{*}\right)_{k, l} \\
& =\sum_{j=1}^{n} p_{j}(t) \sum_{s=1}^{n}\left(\frac{\partial g_{j, k}}{\partial x_{s}}[t] g_{s, l}[t]-\frac{\partial g_{j, l}}{\partial x_{s}}[t] g_{s, k}[t]\right),
\end{aligned}
$$

where $g_{j, k}$ is the $j$-th element of $g_{k}$. Hence for any $k, l \in$ $\{1, \ldots, m\}$,

$\left\langle p(t), \frac{\partial g_{k}}{\partial x}[t] g_{l}[t]-\frac{\partial g_{l}}{\partial x}[t] g_{k}[t]\right\rangle=\left\langle p(t),\left[g_{k}, g_{l}\right][t]\right\rangle=0$. 


\section{Second order conditions on convex poly- topes}

A nonempty subset $U \subset \mathbb{R}^{m}$ is called a convex polytope in $\mathbb{R}^{m}$ if $U$ is an intersection of a finite family of affine half-spaces of $\mathbb{R}^{m}$. Then the adjacent tangent cone $T_{U}^{b}\left(u_{0}\right)$ is equal to the tangent cone of convex analysis to $U$ for any $u_{0} \in U$.

Theorem 4.1 Let $U \subset \mathbb{R}^{m}$ be a convex polytope in $\mathbb{R}^{m}$. Consider a strong local minimizer $(\bar{x}(\cdot), \bar{u}(\cdot))$ of the Mayer problem (3) and $p(\cdot)$ as in the maximum principle of Theorem 2.3. Assume that $\bar{u}(\cdot)$ is integrable on $\left[t_{1}, t_{2}\right]$ for some $0 \leq t_{1}<t_{2} \leq 1$ and (10) holds true. Then $\mathscr{H}_{x u}[t] f_{u}[t]$ is symmetric on the subspace $P_{\bar{u}(t)}$ and $R(t)$ is negative definite on $P_{\bar{u}(t)}$ for a.e. $t \in\left[t_{1}, t_{2}\right]$.

The proof follows the same lines as the proof of Theorem 3.1, but a change of base is involved and the control variations take values in the tangent to $U$ at $\bar{u}(t)$.

The following corollary generalizes the classical Goh condition to boundary controls.

Corollary 4.2 Under all the assumptions of Theorem 4.1 suppose that $f$ is as in (7), where $g(\cdot) \in$ $C^{2}\left(\mathbb{R}^{n} ; M(n \times m)\right)$ and $U$ is a closed convex polytope in $\mathbb{R}^{m}$ and let $g_{k}(x)$ denote the $k-$ th column of $g(x)$. Consider the matrix valued mapping $\Theta:[0,1] \rightarrow M(m \times m)$, whose elements are

$$
\theta_{k, l}(t):=\left\langle p(t),\left[g_{k}, g_{l}\right](\bar{x}(t))\right\rangle \quad \forall k, l \in\{1, \ldots, m\} .
$$

Then for a.e. $t \in\left[t_{1}, t_{2}\right]$, the subspace $\Theta(t)\left(P_{\bar{u}(t)}\right)$ is orthogonal to $P_{\bar{u}(t)}$. In particular, $\Theta(t)$ is equal to zero for a.e. $t \in\left[t_{1}, t_{2}\right]$ such that $\bar{u}(t) \in \operatorname{Int} U$.

Proof. By Theorem 4.1 for a.e. $t \in\left[t_{1}, t_{2}\right], \mathscr{H}_{x u}[t] f_{u}[t]$ is symmetric on $P_{\bar{u}(t)}$. Fix such $t$. Since for all $a, b \in P_{\bar{u}(t)}$, $a^{*}\left(\mathscr{H}_{x u}[t] f_{u}[t]-\left(\mathscr{H}_{x u}[t] f_{u}[t]\right)^{*}\right) b=0$, we get

$$
0=\sum_{k=1}^{m} \sum_{l=1}^{m} a_{k}\left(\mathscr{H}_{x u}[t] f_{u}[t]-f_{u}[t]^{*} \mathscr{H}_{x u}[t]^{*}\right)_{k, l} b_{l} .
$$

Consequently $\left\langle p(t), \sum_{k=1}^{m} \sum_{l=1}^{m} a_{k}\left[g_{k}, g_{l}\right](\bar{x}(t)) b_{l}\right\rangle=0$ and therefore $0=\sum_{k=1}^{m} \sum_{l=1}^{m} a_{k}\left\langle p(t),\left[g_{k}, g_{l}\right](\bar{x}(t))\right\rangle b_{l}=$ $\langle a, \Theta(t) b\rangle$, as claimed.

\section{Second order conditions for smooth con- trol constraints}

In this section we consider a proper closed set $U \subset$ $\mathbb{R}^{m}$ of class $C^{2}$, see for instance [13]. For a strong local minimizer $(\bar{x}(\cdot), \bar{u}(\cdot))$ of the Mayer problem (3) define the following matrix valued mapping $C(\cdot)$ on $[0,1]$

$$
C(t)=\left\{\begin{array}{l}
b_{U}^{\prime \prime}(\bar{u}(t)) \text { if } \bar{u}(t) \in \partial U \\
0 \text { otherwise }
\end{array}\right.
$$

Remark 5.1 If $U$ is of class $C^{2}$, then

a) $\forall u_{0} \in \partial U$ there exists a neighborhood $W\left(u_{0}\right)$ of $u_{0}$ such that the oriented distance $b_{U}(\cdot) \in C^{2}\left(W\left(u_{0}\right)\right)$, see for instance Theorem 4.3 in [13];

b) $\forall u_{0} \in \partial U, T_{U}^{b}\left(u_{0}\right)=\left\{v \in \mathbb{R}^{m}:\left\langle\nabla b_{U}\left(u_{0}\right), v\right\rangle \leq\right.$ $0\}$ and the subspace

$$
P_{u_{0}}:=\left\{v \in \mathbb{R}^{m}:\left\langle\nabla b_{U}\left(u_{0}\right), v\right\rangle=0\right\}=\partial T_{U}^{b}\left(u_{0}\right)
$$

has the dimension equal to $m-1$.

c) $\forall u_{0} \in \partial U, \nabla b_{U}\left(u_{0}\right)$ is the unit outward normal to $U$ at $u_{0}$ and, applying the inverse mapping theorem to $b_{U}$, it is not difficult to show that for any $u \in \partial T_{U}^{b}\left(u_{0}\right)$ we have $-\frac{1}{2}\left\langle b_{U}^{\prime \prime}\left(u_{0}\right) u, u\right\rangle \nabla b_{U}\left(u_{0}\right) \in T_{U}^{b(2)}\left(u_{0}, u\right)$.

For a trajectory-control pair $(\bar{x}(\cdot), \bar{u}(\cdot))$ and for every $t \in[0,1]$ such that $\nabla b_{U}(\bar{u}(t))$ does exist, define the $m-1$ dimensional subspace

$$
S(t):=\left\{u \in \mathbb{R}^{m}:\left\langle\nabla b_{u}(\bar{u}(t)), u\right\rangle=0\right\} .
$$

Theorem 5.2 Let $U$ be of class $C^{2}$. Consider a strong local minimizer $(\bar{x}(\cdot), \bar{u}(\cdot))$ of the Mayer problem (3) and $p(\cdot)$ as in the maximum principle of Theorem 2.3. Assume that for some $0 \leq t_{1}<t_{2} \leq 1, \bar{u}(\cdot)$ is integrable on $\left[t_{1}, t_{2}\right]$.

Then for a.e. $t \in\left[t_{1}, t_{2}\right]$ such that $\bar{u}(t) \in \partial U$ and for some $\delta_{t}>0$

$$
\begin{aligned}
& u^{*}\left(\mathscr{H}_{u u}[s]-\left\|\mathscr{H}_{u}[t]\right\| b_{U}^{\prime \prime}(\bar{u}(s))\right) u=0 \\
& \quad \text { for all } u \in S(s) \text { and for a.e. } s \in\left[t-\delta_{t}, t+\delta_{t}\right] \\
& \text { satisfying }\|\bar{u}(t)-\bar{u}(s)\| \leq \delta_{t} ; \\
& \mathscr{H}_{x u}[\cdot], f_{u}[\cdot] \text { are Lipschitz on }\left[t-\delta_{t}, t+\delta_{t}\right] \\
& \mathscr{H}_{x x}[\cdot], f_{x}[\cdot] \text { are essentially bounded on }\left[t-\delta_{t}, t+\delta_{t}\right]
\end{aligned}
$$

we have $\mathscr{H}_{x u}[t] f_{u}[t]$ is symmetric on the subspace $P_{\bar{u}(t)}$.

\section{Mayer's problem with the endpoint con- straints}

Let $K$ be a proper closed nonempty subset of $\mathbb{R}^{n}$ of class $C^{2}$. Then $b_{K}(\cdot) \in C^{2}$ on a neighborhood of $\partial K$, cf. [13]. We study here the Mayer optimal control problem (1) involving the end point constraints.

Let $(\bar{x}(\cdot), \bar{u}(\cdot))$ be a strong local minimizer of the above problem and $P_{\bar{u}(t)}$ be defined as in Section 2. If $\bar{x}(1)$ belongs to $\operatorname{Int} K$, then the previously developed second order variational analysis does apply as though there were no endpoint constraints. This is due to the fact that small perturbations of $\bar{u}(\cdot)$ lead to feasible trajectories. For this reason we have to investigate only the case $\bar{x}(1) \in \partial K$. We deduce, for instance from [14], the following maximum principle. 
Theorem 6.1 Consider a strong local minimizer $(\bar{x}(\cdot), \bar{u}(\cdot))$ of $(1)$ with $\bar{x}(1) \in \partial K$. Then there exists an absolutely continuous function $p:[0,1] \rightarrow \mathbb{R}^{n}$ and $\lambda \in\{0,1\}, \mu \geq 0$, satisfying $\lambda+\mu>0$, (4), (6) and the transversality condition

$$
-p(1)=\lambda \nabla \varphi(\bar{x}(1))+\mu \nabla b_{K}(\bar{x}(1)) .
$$

The above maximum principle may be abnormal, that is $\lambda=0$. In this case $p(1)$ may be taken equal to $-\nabla b_{K}(\bar{x}(1))$.

Lemma 6.2 Consider a strong local minimizer $(\bar{x}(\cdot), \bar{u}(\cdot))$ of $(1)$ with $\bar{x}(1) \in \partial K$ for which an abnormal maximum principle of Theorem 6.1 holds true for some $\mu>0$. Then for every measurable bounded selection $u(t) \in P_{\bar{u}(t)}$ a.e. in $[0,1]$, the solution $y(\cdot)$ of $(9)$ satisfies $\left\langle\nabla b_{K}(\bar{x}(1)), y(1)\right\rangle=0$ and $\left\langle p(t), f_{u}[t] u(t)\right\rangle=0$ a.e. in $[0,1]$.

If the maximum principle is abnormal, then the cost is not involved into the first order optimality conditions. To apply the variational approach developed in the previous sections, we use the exact penalization which allows to deal with a normal maximum principle.

Definition 6.3 The Mayer problem (1) is called calm at $\bar{x}(\cdot)$ if there exist $\varepsilon>0$ and $M>0$ such that $\forall v \in$ $(0, \varepsilon)$ and $x(\cdot) \in S_{[0,1]}\left(x_{0}\right)$ satisfying $d_{K}(x(1)) \leq v$ and $\|x(\cdot)-\bar{x}(\cdot)\|_{\infty} \leq \varepsilon$ we have $\varphi(x(1)) \geq \varphi(\bar{x}(1))-M v$.

If the Mayer problem (1) is calm at $\bar{x}(\cdot)$, then by Proposition 6.4.3 from [15], there exists $k>0$ such that $(\bar{x}(\cdot), \bar{u}(\cdot))$ is a strong local minimizer of the following minimization problem:

$$
\min \left\{\varphi(x(1))+k d_{K}(x(1)): x(\cdot) \in S_{[0,1]}\left(x_{0}\right)\right\} .
$$

Since $\varphi(\cdot)+k d_{K}(\cdot)$ is no longer $C^{2}$, we need the following (non smooth) maximum principle that can be deduced, for instance, from [14].

Theorem 6.4 Consider a strong local minimizer $(\bar{x}(\cdot), \bar{u}(\cdot))$ of $(11)$ with $\bar{x}(1) \in \partial K$. Then there exists an absolutely continuous function $p:[0,1] \rightarrow \mathbb{R}^{n}$ satisfying (4), (6) and the transversality condition

$$
-p(1) \in \nabla \varphi(\bar{x}(1))+k\left[0, \nabla b_{K}(\bar{x}(1))\right],
$$

where $\left[0, \nabla b_{K}(\bar{x}(1))\right]=\left\{\mu \nabla b_{K}(\bar{x}(1)): \mu \in[0,1]\right\}$.

Denote by $q^{1}(\cdot)$ the solution of (4) for $p(1)=$ $-\nabla \varphi(\bar{x}(1))$ and by $q^{2}(\cdot)$ the solution of (4) for $p(1)=$ $-\nabla \varphi(\bar{x}(1))-k \nabla b_{K}\left(\bar{x}(1)\right.$. Note that $q^{i}(1)$ are extremal points in the transversality condition (12). The mappings $q^{i}(\cdot)$ do not satisfy, in general, the maximum principle (6) but are used below to express the second order necessary optimality conditions. Define for $i=1,2$

$$
\begin{gathered}
\varphi^{1}(\cdot):=\varphi(\cdot), \varphi^{2}(\cdot):=\varphi(\cdot)+k b_{K}(\cdot), \\
\mathscr{H}_{x x}^{i}[\cdot]:=\mathscr{H}_{x x}\left(\cdot, \bar{x}(\cdot), q^{i}(\cdot), \bar{u}(\cdot)\right) .
\end{gathered}
$$

The mappings $\mathscr{H}_{x u}^{i}[\cdot]$ and $\mathscr{H}_{u u}^{i}[\cdot]$ are defined similarly.

Theorem 6.5 Consider a strong local minimizer $(\bar{x}(\cdot), \bar{u}(\cdot))$ of $(11)$ with $\bar{x}(1) \in \partial K$ and $p(\cdot)$ as in Theorem 6.4. Then for every $u(\cdot) \in \mathscr{U}_{A}$ such that $\mathscr{H}_{u}[\cdot] u(\cdot)=$ 0 and the corresponding solution $y(\cdot)$ of (9) verifies $\left\langle\nabla b_{K}(\bar{x}(1)), y(1)\right\rangle=0$, and for any $v(\cdot) \in L^{\infty}\left([0,1] ; \mathbb{R}^{m}\right)$ satisfying $v(t) \in T_{U}^{b(2)}(\bar{u}(t), u(t))$ for a.e. $t \in A$, there exists $i \in\{1,2\}$ such that

$$
\begin{aligned}
0 \geq & \int_{0}^{1}\left(\mathscr{H}_{u}^{i}[t] v(t)+\frac{1}{2} y(t)^{*} \mathscr{H}_{x x}^{i}[t] y(t)\right. \\
& \left.+u(t)^{*} \mathscr{H}_{x u}^{i}[t] y(t)+\frac{1}{2} u(t)^{*} \mathscr{H}_{u u}^{i}[t] u(t)\right) d t \\
& -\frac{1}{2} y(1)^{*}\left(\varphi^{i}\right)^{\prime \prime}(\bar{x}(1)) y(1) .
\end{aligned}
$$

Consider the solution $q(\cdot)$ of the linear system (4) such that $q(1)=-\nabla b_{K}(\bar{x}(1))$ and define the subspaces $\mathscr{T}_{t} \subset$ $P_{\bar{u}(t)}$

$$
\mathscr{T}_{t}:=\left\{u \in P_{\bar{u}(t)}:\left\langle q(t), f_{u}[t] u\right\rangle=0\right\} .
$$

By Lemma 6.2, if the abnormal maximum principle of Theorem 6.1 holds true, then $\mathscr{T}_{t}=P_{\bar{u}(t)}$ a.e. in $[0,1]$ and $q(\cdot)$ satisfies the maximum principle. Observe next that for every measurable essentially bounded selection $u(t) \in \mathscr{T}_{t}$ and the corresponding solution $y(\cdot)$ of (9) we have $\left\langle\nabla b_{K}(\bar{x}(1)), y(1)\right\rangle=0$. Therefore, by Theorem 6.5 , (13) holds true for every $u(\cdot) \in \mathscr{U}_{A}$ such that $u(t) \in \mathscr{T}_{t}$ a.e. in $[0,1]$. This and similar, but lengthly proofs as those of results from Sections 3,4,5 allow to deduce from (13) the following three theorems.

Theorem 6.6 Consider a strong local minimizer $(\bar{x}(\cdot), \bar{u}(\cdot))$ of $(1)$ such that $\bar{x}(1) \in \partial K$. Assume that (1) is calm at $\bar{x}(\cdot)$, that for some $0 \leq t_{1}<t_{2} \leq 1, \bar{u}(\cdot)$ is integrable on $\left[t_{1}, t_{2}\right]$ and for $j=1,2$

$$
\begin{aligned}
& \mathscr{H}_{u u}^{j}[t]=0 \text { a.e. in }\left[t_{1}, t_{2}\right] ; \\
& \mathscr{H}_{x u}^{j}[\cdot], f_{u}[\cdot] \text { are Lipschitz on }\left[t_{1}, t_{2}\right] ; \\
& \mathscr{H}_{x x}^{j}[\cdot], f_{x}[\cdot] \text { are essentially bounded on }\left[t_{1}, t_{2}\right] .
\end{aligned}
$$

Then for a.e. $t \in\left[t_{1}, t_{2}\right]$ with $\bar{u}(t) \in \operatorname{Int} U$ there exists $i \in\{1,2\}$ such that $\mathscr{H}_{x u}^{i}[t] f_{u}[t]$ is symmetric on $\mathscr{T}_{t}$ and

$$
\begin{aligned}
R^{i}(t) & :=\frac{1}{2} f_{u}[t]^{*} \mathscr{H}_{x x}^{i}[t] f_{u}[t]-\frac{1}{2} \frac{d}{d t} \mathscr{H}_{x u}^{i}[t] f_{u}[t] \\
& -\mathscr{H}_{x u}^{i}[t] f_{x}[t] f_{u}[t]+\frac{1}{2} \mathscr{H}_{x u}^{i}[t] \frac{d}{d t} f_{u}[t]
\end{aligned}
$$

is negative definite on $\mathscr{T}_{\text {. }}$. 
Theorem 6.7 Let $U$ be a convex polytope and $(\bar{x}(\cdot), \bar{u}(\cdot))$ be a strong local minimizer of (1) such that $\bar{x}(1) \in \partial K$. Assume that (1) is calm at $\bar{x}(\cdot)$ and for some $0 \leq t_{1}<t_{2} \leq 1, \bar{u}(\cdot)$ is integrable on $\left[t_{1}, t_{2}\right]$ and (14) holds true for $j=1,2$. Then for a.e. $t \in\left[t_{1}, t_{2}\right]$ there exists $i \in\{1,2\}$ such that $\mathscr{H}_{x u}^{i}[t] f_{u}[t]$ is symmetric on $\mathscr{T}_{t}$ and $R^{i}(t)$ is negative definite on $\mathscr{T}_{t}$.

Corollary 6.8 Under all the assumptions of Theorem 6.7 suppose that $f$ is as in (7), where $g(\cdot) \in$ $C^{2}\left(\mathbb{R}^{n} ; M(n \times m)\right)$ and $U$ is a closed convex polytope in $\mathbb{R}^{m}$ and let $g_{k}(x)$ denote the $k$-th column of $g(x)$. For $i=1,2$ define the matrix valued mappings $\Theta^{i}:[0,1] \rightarrow$ $M(m \times m)$, where

$$
\theta_{k, l}^{i}(t):=\left\langle q^{i}(t),\left[g_{k}, g_{l}\right](\bar{x}(t))\right\rangle \quad \forall k, l \in\{1, \ldots, m\} .
$$

Then for a.e. $t \in\left[t_{1}, t_{2}\right]$ there exists $i \in\{1,2\}$ such that $\Theta^{i}(t)\left(\mathscr{T}_{t}\right)$ is orthogonal to $\mathscr{T}_{t}$.

Consider subspaces $S(s)$ defined as in Section 5.

Theorem 6.9 Let $U$ be of class $C^{2}$ and $(\bar{x}(\cdot), \bar{u}(\cdot))$ be a strong local minimizer of the Mayer problem (1) such that $\bar{x}(1) \in \partial K$. Assume that (1) is calm at $\bar{x}(\cdot)$ and for some $0 \leq t_{1}<t_{2} \leq 1, \bar{u}(\cdot)$ is integrable on $\left[t_{1}, t_{2}\right]$.

Then for a.e. $t \in\left(t_{1}, t_{2}\right)$ such that $\bar{u}(t) \in \partial U$ and for some $\delta_{t}>0$ and $j=1,2$ the following relations are satisfied:

$$
\begin{aligned}
& u^{*}\left(\mathscr{H}_{u u}^{j}[s]-\left\|\mathscr{H}_{u}^{j}[s]\right\| b_{U}^{\prime \prime}(\bar{u}(s))\right) u=0 \forall u \in \mathscr{T}_{s} \\
& \text { and a.e. } s \in\left(t-\delta_{t}, t+\delta_{t}\right) \text { with }\|\bar{u}(t)-\bar{u}(s)\| \leq \delta_{t} ; \\
& \mathscr{H}_{x u}^{j}[\cdot], f_{u}[\cdot] \text { are Lipschitz on }\left(t-\delta_{t}, t+\delta_{t}\right) ; \\
& \mathscr{H}_{x x}^{j}[\cdot], f_{x}[\cdot] \text { are essentially bounded on }\left(t-\delta_{t}, t+\delta_{t}\right)
\end{aligned}
$$

we can find $i \in\{1,2\}$ for which $\mathscr{H}_{x u}^{i}[t] f_{u}[t]$ is symmetric on $\mathscr{T}_{t}$.

Remark 6.10 Similar results can be obtained when $K$ is an intersection of a finite family of closed sets $K_{j}$ of class $C^{2}$. Then the assumptions and statements will involve Hamiltonians $\mathscr{H}^{j}$ for each active index $j$.

\section{Conclusions and future works}

For a nonlinear Bolza optimal control problem we proposed pointwise second order necessary optimality conditions extending those of Goh to the case of a closed control constraint given by a polytope or by a $C^{2}$ subset of $\mathbb{R}^{m}$ and for integrable optimal controls that may take values on the boundary of control constraints. Furthermore, we have shown that on the interior of the control constraints Goh conditions hold true for integrable optimal controls without imposing any structural properties on the control set.
This was done by investigating first the free endpoint problem and then, the calmness assumption allowed us to reduce the Mayer problem with endpoint constraints to the one without them, but involving a nondifferentiable cost function. For this equivalent problem we obtained similar pointwise conditions using the developed variational approach for the free endpoint problems. Our future work will concern a larger class of end-point constraints and more general control sets.

\section{References}

[1] B. Bonnard and M. Chyba, Singular trajectories and their role in control theory, Springer, New York, 2012.

[2] B. S. Goh, Optimal singular rocket and aircraft trajectories, Chinese Control and Decision Conference CCDC 2008, Yantai, Shandong, China, pp. 1531-1536.

[3] H. Schättler and U. Ledzewicz, Geometric optimal control, Springer, New York, 2012.

[4] B. S. Goh, Necessary conditions for singular extremals involving multiple control variables, SIAM J. Control, vol 4, 1966, pp. 716-731.

[5] A. J. Krener, The high order maximal principle, in Geometric Methods in Systems Theory, 1973, pp. 174-183.

[6] A. J. Krener, The high order maximal principle and its application to singular extremals, SIAM J. Control Optimization, vol. 15, 1977, pp. 256-293.

[7] A. A. Agrachev and Y. L. Sachkov, Control theory from the geometric viewpoint, Springer-Verlag, 2004.

[8] A. A. Agrachev and R. V. Gamkrelidze, The principle of second order optimality for time-optimal problems, Mat. Sb. (N.S.), vol. 100(142), 1976, pp. 610-643, (648).

[9] M.S. Aronna, J.F. Bonnans, A. V. Dmitruk and P.A. Lotito, Quadratic order conditions for bang-singular extremals, Numer. Algebra Contr. Optim., special issue in honor of Helmut Maurer, vol. 2, 2012, pp. 511-546.

[10] M.S. Aronna, Singular solutions in optimal control: second order conditions and a shooting algorithm, Preprint, 2012.

[11] L. S. Pontryagin, V. G. Boltyanskii, R. V. Gamkrelidze, E. F. Mishchenko, The mathematical theory of optimal processes. Interscience Publishers John Wiley \& Sons, Inc., New York-London, 1962.

[12] D. Hoehener, Variational approach to second-order optimality conditions for control problems with pure state constraints, SIAM J. Control, vol. 50, 2012, pp. 11391173.

[13] M. C. Delfour and J.-P. Zolésio, Shapes and geometries, Society for Industrial and Applied Mathematics (SIAM), Philadelphia, PA, 2011.

[14] R. B. Vinter, Optimal control, Birkhäuser Boston Inc., 2000.

[15] F. H. Clarke, Optimization and nonsmooth analysis, Society for Industrial and Applied Mathematics (SIAM), Philadelphia, PA, 1990. 